\title{
RELAX-based Autofocus Algorithm for High-Resolution Strip-map SAR
}

\author{
Hirad Ghaemi and Mats Viberg \\ Department of Signals and Systems \\ Chalmers University of Technology \\ Gothenburg, Sweden
}

\author{
Frank Gekat \\ SELEX Sistemi Integrati GmbH \\ Neuss, Germany
}

\author{
Michele Galletti and Thomas Boerner \\ Microwave and Radar Institute \\ DLR, German Aerospace Center \\ Wessling, Germany
}

\begin{abstract}
This paper addresses the non-iterative quality phase gradient autofocus (QPGA) technique, which was originally proposed to remove one-dimensional phase errors in spotlightmode synthetic aperture radar (SAR) imagery. By enriching the source pool, the method is modified in a way suitable for autofocus in stripmap-mode SAR system with the advantage of being independent of any priori assumptions. Unlike the QPGA the potential candidates, i.e., dominant scatterers located along azimuth in each specific range bin, are automatically selected by exploiting the one-dimensional RELAX algorithm. Furthermore, RELAX is capable to estimate the size of blur window which is, in fact, associated with the Doppler spread of signal spectrum. The corresponding model includes four parameters i.e., complex amplitude, delay, Doppler center and spectral width. The proposed method has been applied to data extracted by a ground-based rotating coherent Doppler radar operating in strip-mapping mode SAR, with the aim of highresolution clutter detection.
\end{abstract}

\section{INTRODUCTION}

Of several autofocus techniques suggested to mitigate the one-dimensional phase error, along-track dependent phase error repetitive over all range bins, the phase gradient autofocus (PGA)[1,2] has been found to be robust for both spotlight and stripmap SAR. However, at most one dominant scatterer of each range bin is used in this algorithm to determine the error function, whereas the other strong and high-quality scatterers, which might be available in that range bin, are entirely discarded. This can be a serious issue in estimating the phase error involved in stripmap SAR, in which each and every single scatterer has only a partial contribution to estimation of the blur function. Furthermore, the error in low-content images, especially those with long aperture having a small number of dominant scatterers, sparsely and discretely populated over range swath, can not be effectively removed due to the lack of sources necessary to cover the whole aperture in process. In other words, after several iterations there exist some segments of phase error, which still remain undetermined.

To tackle this problem to some extent, the QPGA [3]

This work was supported by SELEX-SI Gematronik Inc.and DLR (German Aerospace Center). comes into play. In fact, this method enriches the source pool to increase the possibility of full phase error measurements by taking more than one high-quality scatterer into autofocus analysis in each range bin. The quality of scatterers is to be investigated through their corresponding normalized variance in aperture domain prior to the selection process according to the QPGA. Since the reported QPGA is only suggested for the autofocus of spotlight mode SAR, some modifications must be made to apply it to the stripmap mode SAR. To realize this, one can easily follow the steps described in [2] for all scatterers, even those within the same range.

Despite the robustness of the QPGA, there are a couple of a priori assumptions, which can restrict the maximum achievable performance and therefore must be disregarded or modified if high resolution is demanded. The first assumption is the number of potential candidates, decided to be a value between three and six according to some empirical experiments which might not be true in general. One can turn to the RELAX algorithm [4,5], which is a robust spectral estimation algorithm used for feature extraction and spectrum estimation, to automatically find out the maximum number of dominant scatterers and their corresponding parameters based on the generalized Akaike information criterion (GAIC) [4]. This algorithm is flexible in a sense that a simple amplitude threshold may be set both to limit the selected dominant scatterers to those with a desired signal to noise ratio (SNR) level and also to accelerate the quite long iterative process inherent to the algorithm as well. Since the normalized variance, or equivalently contrast, of a scatterer is directly related to the Doppler spread of that scatterer normalized to the pulse repetition frequency (PRF) in image domain, the high contrast scatterers can be selected and isolated readily throughout the spectral estimation by RELAX. Therefore, the steps associated with quality-checking in QPGA are entirely avoided.

The second assumption is made about the window size by the QPGA algorithm. In the corresponding paper, this size is basically considered to be fixed for all candidates. Having exploited the RELAX algorithm for feature extraction of scatterers, the size of window is determined independently and 
directly from the estimated Doppler spread for each and every candidate. Thus the two key steps, i.e. selection of brightest scatterers and their corresponding window size, are performed jointly and automatically via the RELAX algorithm without any necessary assumptions at the beginning of this new noniterative strip-map SAR autofocus technique.

The details of the proposed algorithm are going to be described in the next section. In the third section, a couple of experimental results are demonstrated to prove the versatility of this algorithm in resolution enhancement of a ground-based rotating SAR system whose data has incurred an unknown phase error given rise to by several reasons such as aperturedependent phase error, uncompensated motion of platform or its motion perturbation and propagation path distortion or propagation anomalies in general. Finally some concluding remarks are stated at the last section of this paper.

\section{Methodology}

Since parameter estimation using the one-dimensional (1D) RELAX algorithm turns out to be the most consequential part of this new strip-map autofocus algorithm, it is worth investigating some details of this algorithm prior to further description of autofocus SAR. The measured samples along aperture $y(m)$ can be expressed as

$$
y(m)=s(m)+v(m) \quad, \quad m=0,1, \ldots, M-1
$$

where $v(m)$ are the samples of noise and $s(m)$ are the model-based samples given as

$$
\begin{gathered}
s(m)=\sum_{k=1}^{K} \alpha_{k} g_{k}(m) \\
g_{k}(m)=\exp \left(j 2 \pi P R I \cdot f_{k} m\right) \\
\times \exp \left(-\frac{\pi^{2}}{4 \ln 2}\left[P R I \cdot \Delta_{k} \cdot\left(m-d_{k}\right)\right]^{2}\right)
\end{gathered}
$$

PRI is the pulse repetition interval, which denotes the sampling interval in the aperture domain, indeed. $K, \alpha_{k}, f_{k}$, $\Delta_{k}$, and $d_{k}$ are the number of scatterers, complex amplitude, Doppler centroid, Doppler spread and proper delay of the $k$ th scatterer, respectively. The goal is to estimate these unknown parameters representing the features of the target(s) through minimizing the nonlinear least squared (NLS) cost function $C_{1}$ which is

$$
C_{1}\left(\left\{\alpha_{k}, f_{k}, \Delta_{k}, d_{k}\right\}_{k=1}^{K}\right)=\|\mathbf{y}-G \boldsymbol{\alpha}\|^{2}
$$

where

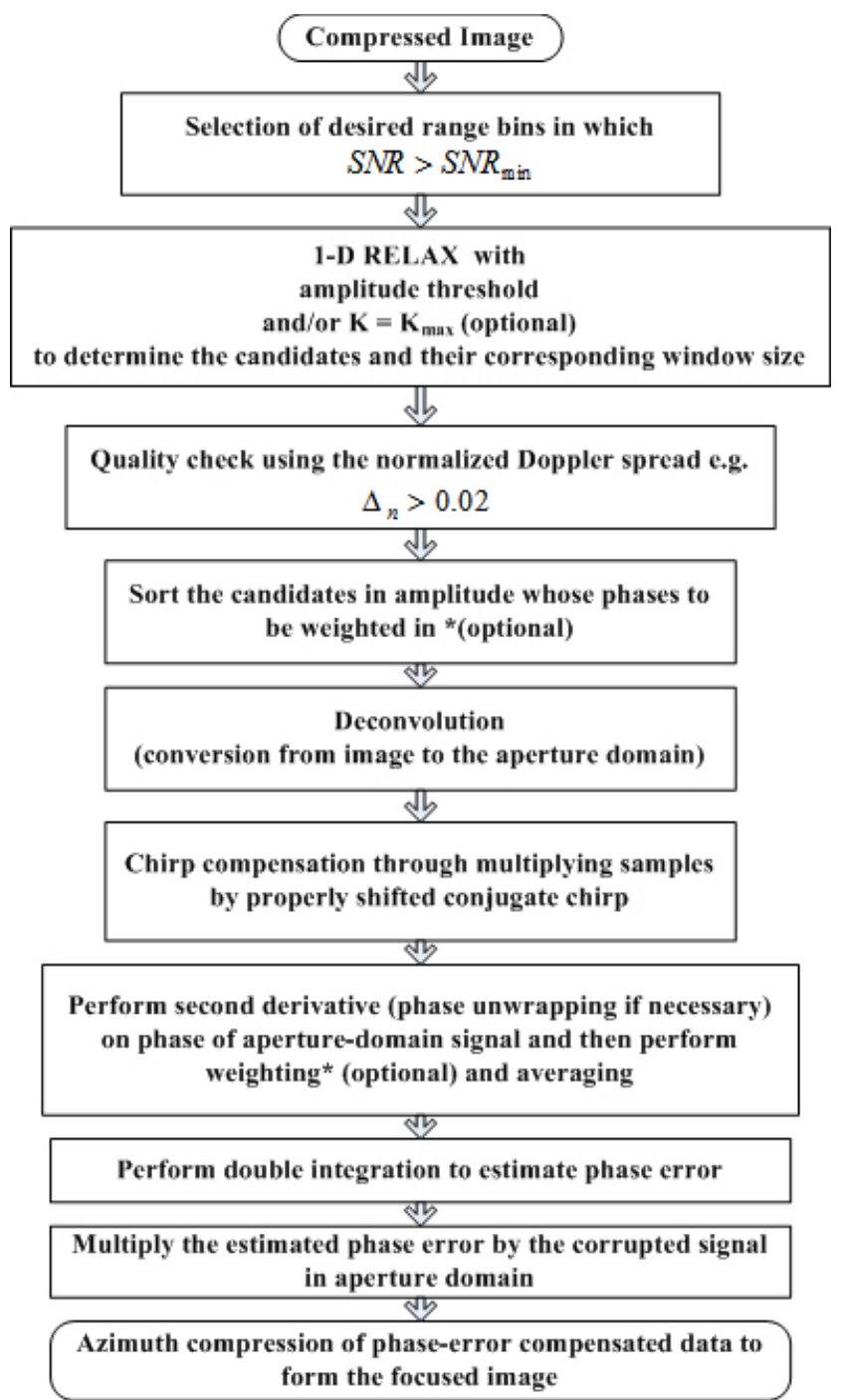

Figure 1. Block diagram of the RELAX-based autofocus algorithm in stripmap SAR

$$
\begin{gathered}
\mathbf{y}=\left[\begin{array}{llll}
y(0) & y(1) & \cdots & y(M-1)
\end{array}\right]^{T} \\
\boldsymbol{\alpha}=\left[\begin{array}{llll}
\alpha_{1} & \alpha_{2} & \cdots & \alpha_{K}
\end{array}\right]^{T} \\
G=\left[\begin{array}{llll}
\mathbf{g}(0) & \mathbf{g}(1) & \cdots & \mathbf{g}(M-1)
\end{array}\right]^{T} \\
\mathbf{g}(m)=\left[\begin{array}{llll}
g_{1}(m) & g_{2}(m) & \cdots & g_{K}(m)
\end{array}\right]
\end{gathered}
$$

" $T$ " and $\|\cdot\|$ denote the matrix transpose and Euclidean norm respectively. Note that the vectors are depicted in bold letters, and that (4) is a non-linear function of $f_{k}, \Delta_{k}$, and $d_{k}$, but linear in $\alpha_{k}$. Thus, rather than trying to solve for all 
parameters simultaneously, an iterative approach is used, where the parameters of one scatterer are considered at the time. Assume the parameters of all scatterers but the $k$ th are known (or previously estimated). .Minimizing $C_{1}$ with respect to $\alpha_{k}$ then gives its estimate $\hat{\alpha}_{k}$ which is

$$
\widehat{\alpha}_{k}=\frac{G_{k}^{H} \mathbf{y}_{k}}{G_{k}^{H} G_{k}}
$$

" $H$ " stands for Hermitian matrix operation and “^” from now on denotes estimates associated with the four desired parameters. In equation (9), $G_{k}$ is the $k$ th column of matrix $G$, and $\mathbf{y}_{k}$ is calculated as follows

$$
\mathbf{y}_{k}=\mathbf{y}-\sum_{i=1, i \neq k}^{K} \alpha_{i} G_{i}
$$

By inserting equation (9) into equation (4) and performing some manipulation in an attempt to minimize $C_{1}$, the following new cost function $C_{2}$ is to be maximized to obtain the three parameters $f_{k}, \Delta_{k}$, and $d_{k}$

$$
C_{2}\left(f_{k}, \Delta_{k}, d_{k}\right)=\frac{\left|G_{k}^{H} \mathbf{y}_{k}\right|^{2}}{G_{k}^{H} G_{k}}, k=1,2, \ldots, K
$$

The maximization of (10) requires a three-dimensional search over the unknown parameters. Following a procedure similar to $[4,5]$ the Doppler frequency is readily estimated by localizing the argument of dominant peak in 1-D fast Fourier transform(FFT), with sufficient zero padding for the sake of precision, while fixing the other parameters. Thus

$$
\widehat{f}_{k}=\arg \max \left\{F F T\left\{\frac{\left\|\operatorname{Mult}\left(G_{k 0}, \mathbf{y}_{k}\right)\right\|^{2}}{G_{k 0}^{H} \cdot G_{k 0}}\right\}\right\}
$$

in which $G_{k 0}$ is $G_{k}$ at $f_{k}$ equal to zero. "Mult $(x, y)$ " is defined as element-by-element multiplication of two vectors $x$ and $y$ with equal length. Using (12), both the Doppler spread and delay can be iteratively determined via a simple 2-D search to maximize (11).

The following is the summary of all steps according to [4] excluding the steps related to the noise model.

Assume $K=1$. Determine the first estimate of four parameters of the first dominant scatterer from $\mathbf{y}$,i.e. $\left\{\hat{\alpha}_{1}, \widehat{f}_{1}, \widehat{\Delta}_{1}, \widehat{d}_{1}\right\}$ as described above.

Assume $K=2$. Compute $\mathbf{y}_{2}$ with equation (10) by using estimated parameters calculated in step (1). From that, the

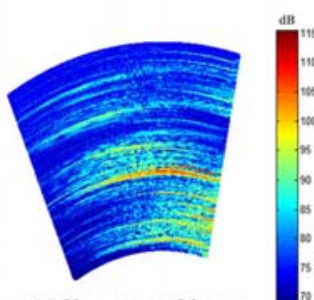

(a) Unprocessed image

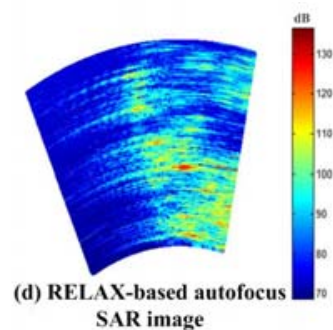

SAR image
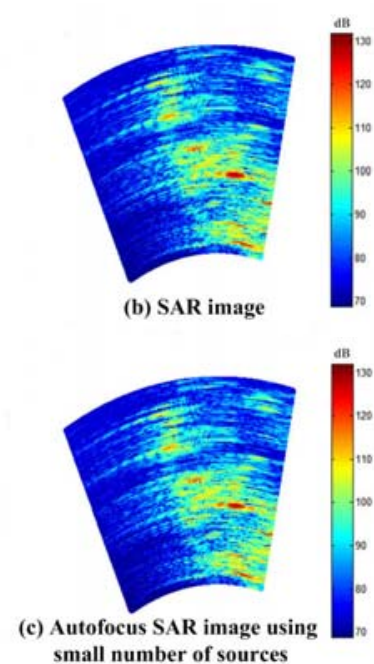

small number of sources
Figure 2. Raw, SAR, and autofocused SAR images

parameters $\left\{\hat{\alpha}_{2}, \hat{f}_{2}, \widehat{\Delta}_{2}, \widehat{d}_{2}\right\}$ of the second scatterer are obtained. Next, compute $\mathbf{y}_{1}$ with (10) by using these recently estimated parameters and then redetermine $\left\{\hat{\alpha}_{1}, \widehat{f}_{1}, \widehat{\Delta}_{1}, \widehat{d}_{1}\right\}$ from $\mathbf{y}_{1}$.Continue this iterative procedure until the practical convergence (explained later) occurs.

Assume $K=3$. Calculate $\mathbf{y}_{3}$ with equation (10) by taking $\left\{\widehat{\alpha}_{i}, \widehat{f}_{i}, \widehat{\Delta}_{i}, \widehat{d}_{i}\right\}_{i=1}^{2}$ estimated at the end of the previous step. Obtain $\left\{\hat{\alpha}_{3}, \hat{f}_{3}, \widehat{\Delta}_{3}, \hat{d}_{3}\right\}$ from $\mathbf{y}_{3}$ as before. Next, compute $\mathbf{y}_{1}$ by using $\left\{\hat{\alpha}_{i}, \hat{f}_{i}, \widehat{\Delta}_{i}, \hat{d}_{i}\right\}_{i=2}^{3}$ via equation (10). Similarly compute $\mathbf{y}_{2}$ with equation (10) by using $\left\{\hat{\alpha}_{i}, \widehat{f}_{i}, \bar{\Delta}_{i}, \widehat{d}_{i}\right\}_{i=1,3}$. Repeat these three substeps until the practical convergence is achieved.

For $K>3$ continue similarly until $K=\mathrm{K}$, which is the integer that minimizes the following GAIC cost function.

$$
G A I C_{K}=M \ln \left(\|\mathbf{e}\|^{2}\right)+4 \ln (\ln M) \cdot(3 K+1)
$$

where the error vector $\mathbf{e}$ is defined as

$$
\mathbf{e}=\mathbf{y}-\sum_{k=1}^{K} \alpha_{k} G_{k}
$$

Practical convergence in the above iterative process can be determined by checking the relative change in the cost function $C_{1}$ in equation (4) between two consecutive iterations to make sure that $C_{1}$ gets less than a certain value, for instance $10^{-4}$. Here, as brought up earlier, another threshold regarding minimum tolerable SNR is also set to limit the maximum number of selected scatterers, $\mathrm{K}$, and the 

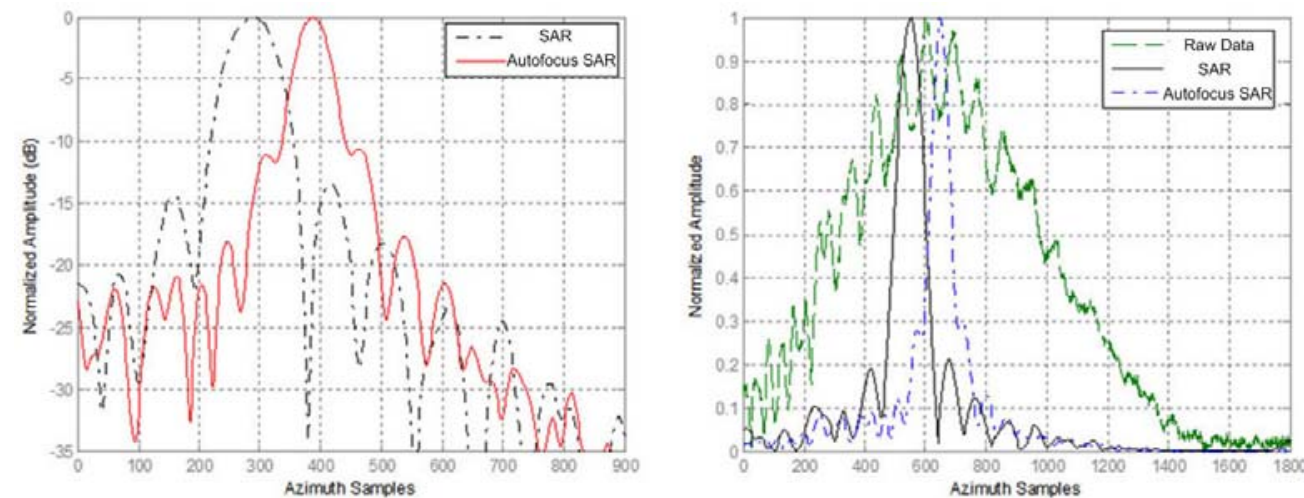

Figure 3. Demonstration of improvement in along-track resolution via RELAX-based autofocus algorithm

number of steps as well prior to GAIC criterion while updating $K$.

By doing this, not only the scatterers with fairly good brightness are selected, but also the speed of the algorithm increases. On the other hand, one may force the algorithm to terminate the iteration when $\mathrm{K}$ reaches a certain maximum value to circumvent collecting unnecessary candidates having no serious impact on the final result except adding complexity to the SAR processing of those scenes, which contain densely populated high-featured targets.

All the steps of this RELAX-based autofocus algorithm are summarized in the block diagram demonstrated in Fig.1. The along-track resolution of a compressed image, assumed to be not fully focused, is intended to become improved after passing through this autofocus algorithm. Before starting with the first and the most important step, i.e., parameter extraction via RELAX, it is going to be much faster as well as more efficient to leave those low-energy range bins out of the analysis. This can be readily taken care of by setting a minimum SNR at the beginning. Next, the strong potential candidates are automatically selected, isolated and windowed using RELAX. The window size of the $k$ th scatterer $W_{k}$ is determined by employing its estimated 3-dB window size $\Delta_{k}$ (based on Gaussian model in (3)) as below

$$
W_{k}=\beta \frac{P R F}{\Delta_{k}} \quad, \quad \beta \geq 1
$$

$P R F$ is pulse repetition frequency, inverse of $P R I$. The factor $\beta$ should be chosen to be a value around two if one considers $10-\mathrm{dB}$ bandwidth as the authors did in [1] for calculating the window size at the first iteration. The quality of candidates is investigated through checking of their associated Doppler width normalized to $P R F$, i.e., $\Delta_{n}$. In this paper, a threshold of 0.02 is considered. This will partly guarantee to simply bring those point-like targets, less interfered with others of different kinds, into the desired source pool. This quality check, however, does not play a crucial role in this autofocus algorithm.

At this stage, one may sort the collected candidates in descending order of their corresponding amplitudes whose respective phases in the aperture domain might be weighted by using window function such as a hamming window. The purpose of this optional step is to put more emphasis on the contribution of strong scatterers than on that of the weak ones to the estimation process of the induced phase error.

From now on, the steps are fairly similar to those explained in [2]. To reconstruct the range-compressed data, aperture domain data, these windowed candidates must be deconvolved with an appropriate FM chirp. In other words, the conjugate of the chirp function employed for cross-range compression is to be convolved with the collected data samples. To yield aperture samples consisting of the target dependent complex constant multiplied by a complex exponential associated with the phase error, the chirp phase (phased modulation, PM) must be removed from the phase of gathered sources in rangecompressed domain. This is simply accomplished through multiplication of each and every single candidate by the conjugate of the chirp signal properly shifted according to the corresponding position of the candidate. Since each candidate spans a particular set of pulses during its SAR illumination, the source pool produces a set of displaced apertures each of which reveals only a specific segment of the phase error function. As justified in [2], the second difference of phase is computed instead of the phase gradient and then being averaged across the target apertures which overlap a certain part. Herein, the weighting (or equivalently windowing) might be performed prior to averaging in case the scatterers are already sorted. The phase error function is finally estimated by applying the double integration. Note that the phase unwrapping process turns out to be necessary in dealing with the phase of sources obtained in aperture domain. It is imperative that the phase is unwrapped throughout the calculation of the phase differences and also before double integration is carried out; otherwise it will result in a wrong estimation of the phase error.

To accomplish the phase unwrapping, the fast and simple approach reported in [6] is found to be handy and therefore it is applied to the current problem.

At the end, the range-compressed samples in aperture domain are corrected by the conjugate of the phase error before the azimuth compression is performed.

\section{EXPERIMENTAL RESULTS}

In this section, a couple of examples are presented to verify the performance of the RELAX-based autofocus SAR 
algorithm. The radar system, provided by SELEX-SI

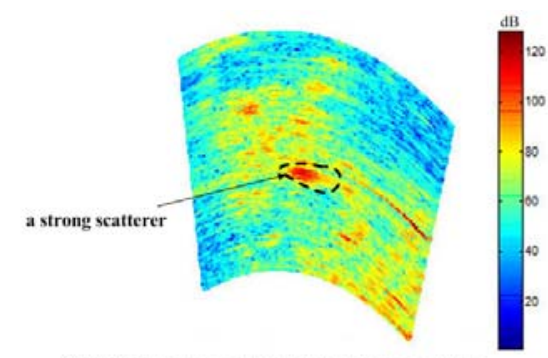

(a) SAR image corrupted by phase error

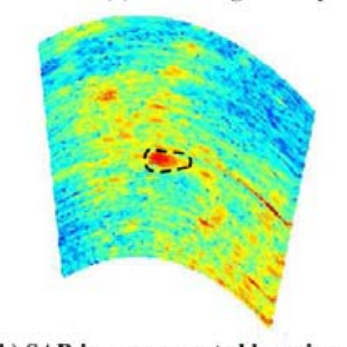

(b) SAR image corrected by using [2] with only one iteration

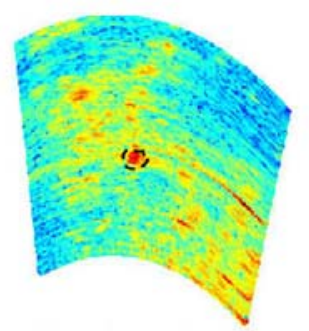
based autofocus algorithm (c) SAR image corrected by RELAX

Figure 4. Focused images by RELAX and [2]

Gematronik Inc., is a ground-based rotating Doppler weather radar carrying a small antenna with a very low along-track resolution [7]. In terms of SAR technology the system is a ground-based circular SAR (CSAR) operating in the stripmapping mode. The data of a few images, referred later on, are extracted by this radar in clear air. Thus the very bright pixels in the images simply exhibit ground clutter. The goal is to increase the along-track resolution to a great extent in order to gain high-resolution images from clutter. The resultant resolution can easily be shown to be comparable to that of a very big antenna operated by Gematronik and DLR (German Aerospace Center) [7]. In the first example, illustrated in Fig.2, we aim at removing the existing phase error arisen probably from several sources, either internal ones, i.e. those by platform, aperture, etc., or the external ones, due to e.g. propagation anomalies specifically at far distances.

The raw image, unprocessed image, and compressed image obtained by strip-map CSAR processing in [7], are shown in Fig.2 (a) and Fig.2 (b), respectively. Restricting the maximum number of candidates to 4 per range bin in the RELAX algorithm will result in a little improvement in alongtrack resolution as opposed to the one in which the number of scatterers are determined by the algorithm itself. The corresponding results are depicted in Fig2 (c) and Fig.2 (d), respectively. In some range bins, the number of dominant scatterers, or let's say strong point scatterers, has been found to exceed the value of six. The maximum image intensity, stated near color bars on top, implies the processing gain achieved by SAR matched filter and eventually by the autofocus algorithm.

The amount of increase in processing gain introduced by the RELAX autofocus (see Fig.2 (d)) is about $6 \mathrm{~dB}$. To perceive the final enhancement even better, the normalized azimuth pattern of raw samples, SAR samples, and RELAX- based autofocus SAR samples in a specific range gate are presented in Fig.3. Moreover, the associated results of SAR and autofocus SAR are also illustrated in $\mathrm{dB}$ for the sake of contrast between their corresponding side-lobe levels. Note that none of the SAR images presented in this paper are windowed as it is commonly done for mitigating the side lobes. The reason is to show the maximum possible resolution which is not being degraded by window effect.

Herein, another example is taken into consideration in order to compare the performance of this non-iterative RELAX-based autofocus algorithm with that of strip-map autofocus with only one iteration reported in [2]. A sinusoidal phase error is artificially injected into the raw data whose SAR image is illustrated in Fig.4 (a). In this figure, a dash-line curve encircles a few pixels representing a strong scatterer or a group of small and bright scatterers. Following the autofocus method in [2] and RELAX autofocus algorithm, one will end up with SAR images depicted in Fig.4 (b) and Fig.4(c) , respectively. The available number of dominant sources, those with good quality and high SNR level, exceeds ten in most of the range bins.

\section{CONCLUSIONS}

In this paper a new non-iterative autofocus algorithm has been proposed for improvement of along-track resolution in strip-map SAR imagery. The 1-D RELAX spectral estimation method is involved in this algorithm to automatically perform two key steps which are the selection of strong as well as high-quality sources in each range bin and also their corresponding window size, used to capture the blur function without recourse to any a priori assumptions. A unique experiment with ground-based rotating Doppler radar has been conducted and a couple of their corresponding SAR images have been demonstrated to verify the performance of this algorithm. For low-content images, in which dominant and/or point-like scatterers are mainly distributed along azimuth rather than over various range bins, the RELAX autofocus algorithm can be a better alternative than its counterpart [2]. On the other hand, if the aforementioned thresholds are not set, the RELAX procedure might considerably slow down the whole autofocus process for those images with densely populated strong/high quality scatterers almost available all over the image. In short, it can be deduced that this algorithm aims at taking full advantage of all available good sources to make a decent estimation of the phase error function without any iteration.

\section{REFERENCES}

[1] D. E. Wahl, P. H. Eichel, D. C. Ghiglia, and C. V. Jakowatz, "Phase gradient autofocus-A robust tool for high resolution SAR phase correction,'” IEEE Trans. Aerosp. Electron. Sys., vol. 30,pp. 827-834, July 1994.

[2] D. E. Wahl and C. V. Jakowatz, "New approach to strip-map SAR autofocus," in Proc. IEEE $6^{\text {th }}$ Digital Signal Processing Workshop, 1994, pp. 53-56.

[3] H. L. Chan and T. S. Yeo, "Noniterative quality phase-gradient autofocus (QPGA) algorithm for spotlight SAR imagery,” IEEE Trans. Geosci. Remote Sens., vol. 36, pp. 1531-1539, September 1998.

[4] J. Li and P. Stoica, "Efficient mixed-spectrum estimation with application to target feature extraction," IEEE Trans. Signal Processing, vol. 44, pp. 281-295, February 1996. 
[5] Z. S. Liu and J. Li, "Feature extraction of SAR targets consisting of trihedral and dihedral corner reflectors," IEE Proc. Radar, Sonar Navig., vol. 145, pp. 161-172, June 1998.

[6] M. D. Pritt and J. S. Shipman, "Least-squares two-dimensional phase unwrapping using FFT's,” IEEE Trans. Geosci. Remote Sens., vol. 32, pp. 706-708, May 1994.
[7] H. Ghaemi, M. Galletti , T. Boerner, F. Gekat, and M. Viberg, "Synthetic aperture weather radar," M.Sc. Thesis , DLR (German Aerospace Center), Wessling, July 2008. 